\title{
An Extensible Platform for Interactive, Entertaining Social Experiences with an Animatronic Character
}

\author{
Sabrina Haskell \\ Entertainment Technology \\ Center \\ Carnegie Mellon University \\ 700 Technology Dr., Rm. 5305 \\ Pittsburgh, PA 15219 \\ shaskell@andrew.cmu.edu
}

\author{
Andrew Hosmer \\ Entertainment Technology \\ Center \\ Carnegie Mellon University \\ 700 Technology Dr., Rm. 5305 \\ Pittsburgh, PA 15219
}

\author{
Eugenia Leu \\ Entertainment Technology \\ Center \\ Carnegie Mellon University \\ 700 Technology Dr., Rm. 5305 \\ Pittsburgh, PA 15219 \\ eyj@andrew.cmu.edu
}

\begin{abstract}
The fields of human-robot interaction and entertainment robotics are truly interdisciplinary, combining the best of computer science, psychology, and mechanical engineering. However, they are areas which have thus far been largely limited to technical specialists, excluding animators, writers, and other artists from the development of interactive content. We attempt to open these fields to non-technologists through an easily extensible platform for the rapid development of social interactions between humans and animatronic characters. Specifically, the Interbots Platform consists of 1) a servo-driven animatronic character named Quasi and 2) a suite of tools to author decisions, animate the character, and design complete, interactive, entertaining social experiences. We have created a graphical editor to author behaviors for the character, as well as extensions for Alias Maya and Macromedia Director, empowering artists already familiar with these applications to create animatronic-enabled content. The usability of the Interbots Platform was verified when interdisciplinary student teams of artists and computer scientists were able to create engaging entertainment experiences with Quasi in two-week development cycles.
\end{abstract}

\section{INTRODUCTION}

The past past five years have seen an explosion in the consumer robotics industry. With over one million units sold since their introduction in 2002, iRobot's Roomba vacuum cleaner has paved the way for a robotic presence in the home [8]. Realizing that consumers crave entertainment as much as (if not more than) amenities, companies began introducing entertainment robots into the market. The popularity of Sony's AIBO dog and Wow Wee Toys' Robosapien have demonstrated that entertainment robotics is a viable industry.

Unlike the traditional linearly pre-scripted show animatron- ics found in many theme and amusement parks, the novelty of household entertainment robots is in their interactivity. Sony's AIBO, for example, has the ability to locate specific objects, recognize its owner's voice, and sense when it is being touched. The robotic dogs are capable of "feeling" six emotions, and are shipped with a default personality that changes over time as their owners interact with them. The latest versions of AIBO feature official support for wireless remote control as well as the ability to download small personality additions such as games and sounds. More advanced features such as total personality make-overs and the ability to create custom interactive games are not officially supported. Ambitious hobbyists may utilize official and third party software developer kits to fully customize their AIBO, however, these kits require knowledge of lowlevel programming concepts.

The Interbots Initiative research group at the Entertainment Technology Center (ETC) seeks to leverage the success of household entertainment robotics and the interest of the hobbyist community to introduce interactivity to the field of higher-end custom animatronics. We also intend to give non-technologists the ability to develop content for interactive animatronic experiences. These objectives will be accomplished through two main goals: 1) Develop a fully interactive, autonomous animatronic character that is believable and entertaining, and 2) Develop an extensible platform that allows artists and other non-technologists to rapidly design and create complete, interactive, and entertaining social experiences with animatronic characters. We feel that an embodied character is critical to a believable animatronic experience- no matter how impressive the technology, it is impossible to suspend an audience's disbelief and create the illusion of life without an engaging character and personality. We also believe that the most successful entertainment experiences involve the contributions of writers, animators, sculptors, painters, and other artists. Therefore, the development of simple, intuitive content creation tools that allow non-technologists to author content for entertainment robotics is essential.

In accordance with these goals, we have created a believable animatronic character named Quasi, and an extensible platform that allows for the development of interactive social experiences with Quasi. Interactivity designers need not rely on pre-manufactured content; the Interbots Platform al- 
lows new, experiences to be developed quickly and easily. No programming or technical knowledge is required-developers can create new behaviors for the animatronic character using simple custom graphical authoring tools. These tools allow for Quasi's interactive experiences to range from fully autonomous to completely pre-scripted. The usability of the authoring tools was verified when teams of four students with no previous knowledge of the Interbots Platform were able to develop a theatrical karaoke show, an interactive photo kiosk, a live puppeteering interface, and interactive dream sequences in two week development periods.

The remainder of this paper discusses the various components of the Interbots Platform and the content authoring process. Section 2 briefly reviews related work in the field of animatronics. The design and construction of the Platform's hardware is discussed in Section 3. Section 4 describes the figure's control software and the content authoring tools. Several examples of rapidly developed interactive, entertainment experiences are presented in Section 5. The future direction of this research is discussed in Section 6, and conclusions are presented in Section 7.

\section{RELATED WORK}

This project builds on previous work by Carnegie Mellon University's Entertainment Technology Center students, but significantly extends and expands upon it. The ETC's Interactive Animatronics Initiative began with the Doc Beardsley Project [3] which sought to develop technology that enables animatronic figures to become conversationally interactive. This goal was met by combining a multitude of technologies (speech recognition, synthetic interview, discussion engine, audio, vision, and animatronics), which resulted in a new medium for interactive entertainment. Realizing the limitations of a conversational engine, the renamed Interbots Initiative has refocused on creating an expressive, believable character and developing an extensible platform for the creation of interactive, social experiences with an animatronic robot.

Advancements in animatronic technology differ between academia and industry, with academia concentrating on the creation of artificial intelligence and industry focusing on hardware improvements. Within academia, our approach is related to that of Breazeal et al [6], Pineau et al [9], and Bruce et al [7], whose research with humanoid and animatronic robots explores social aspects of human-robot interaction. Our work is most similar to that of Breazeal et $a l$, whose research with their animatronic robot Leonardo centers around human-robot collaboration and learning [6]. Like Leonardo, our animatronic robot Quasi is influenced by behavioral, emotional, and environmental motivators. However, our focus is on engaging humans in an entertaining experience, and not specifically on task-collaboration or robotlearning. The biggest difference in our approach is that we are not concerned with replicating human intellect and emotional processes- rather, we wish to emulate it. Consequently, our work is influenced by theatre and focuses largely on the character and personality of the animatronic robot. In terms of creating the illusion of a living character, the audience cannot distinguish between a character that "actually thinks like a human" and a character that "appears to think like a human." Instead of focusing on artificial in- telligence research, we have chosen to apply our efforts to mechanisms for creating complete interactive entertainment experiences with an animatronic character.

Our work is also similar to Breazeal et al's Interactive Robot Theatre [5], a robotic terrarium featuring an interactive, anemone-like creature. Our work largely differs in the depth of interactivity and audience participation; the anemone's interactions center around tracking guests' movements and recoiling from guests when frightened. Quasi has voice recognition capabilities, can receive input through the touchscreen on his kiosk, and has the ability to recognize specific individuals through the use of an active login mechanism, allowing for much deeper and more personal interactions.

Within industry, our work is most similar to the animatronics found in Disney Theme Parks. Typically, these have not been interactive, simply playing a linear pre-programmed show. The best of these scripted attractions can exhibit nuanced details of personality and emotion, but are completely indifferent to people and the changing environment around them. Interesting recent developments have begun to appear from the labs of Walt Disney Imagineering Research \& Development, most notably Lucky the Dinosaur [4]. It is believed that Lucky is controlled by the "man-behind-thecurtain" technique, something we hope to explore ourselves via the Guided Performance Interface (GPI) as outlined in the Section 6. In many ways, our extensible platform for interactive animatronics is a bridge between academia and industry.

\section{HARDWARE}

The hardware of the Interbots Platform currently consists of three major components: the animatronic figure, the kiosk in which the figure resides, and the show control hardware. The design and construction process of the animatronic character is detailed in Section 3.1, the kiosk and its many input sensors are described in Section 3.2, and some of the show control hardware used in the Platform is briefly discussed in Section 3.3.

\subsection{Quasi the Robot}

The most visible component of the Interbots Platform is, of course, the physical robot. For our research we have developed a custom-built figure we call "Quasi". Quasi is an expressive anthropomorphic humanoid robot with a slightly masculine appearance. Recent developments have introduced pre-recorded speech, but a substantial amount of his activity is non-verbal, instead relying expressive facial features and gestures.

Having the liberty to custom-design a figure for our purposes, we started our development process in character design. The approach was similar to the one taken for film or video-game characters, but with some notable differences. A blue-sky conceptual phase led to three varied concepts- one of which was a robot in form. Iteration led us to move forward with the robot figure after merging the best qualities from all three designs. Pencil sketches established a design style, which was then fleshed out during the creation of a 3D model in Alias Maya- an industry preferred modeling and animation package. At this point physical constraints became a notable concern. When designing a virtual charac- 
ter, mass, realistic joint sizes, and other mechanical factors do not need to be considered. For animatronic characters, real-world physical limitations require function to supercede form. We paired a traditional 3D artist with the industrial designer who built the robot in order to ensure that the design was both functional and appealing. A copy of the 3D model was imported into SolidWorks, a mechanical design application. The placement of the internal joints, motors, and armatures occurred in SolidWorks parallel to iterations on the cosmetic look of the character in Maya.

Fabrication began once the external cosmetic shell and internal armature had been finalized in virtual space. Engineering drawings from SolidWorks were used to hand-machine the armature, while the shell pieces modeled in Maya were exported and fabricated on a $3 \mathrm{D}$ printer. The pieces from the $3 \mathrm{D}$ printer were not nearly durable nor lightweight enough to use directly on the robot. This problem was solved by making molds of the printed pieces and casting final parts out of a fiberglass-reinforced resin. These parts could stand up to the challenge of being used on the robot, and had the benefit of being a one-to-one match of the 3D Maya model. As discussed in Section 4.2.3, this allows artists to use Maya's animation tools to create lifelike movement for the physical robot.

In terms of physical construction, Quasi has more in common with a radio-controlled model aircraft than with a traditional robot. His armature utilizes a large number (27) of variously-sized hobby servo motors to move. The armature itself is machined out of lightweight aluminum. To facilitate the character's ability to express emotion, ten servos have been dedicated to movements of the eyes and eyelids alone. (In addition to telescoping in and out, each eye has an upper and lower eyelid that can be raised, lowered, and tilted diagonally to emulate the movement of eyebrows.) Hobby servos combine a DC rotational motor, a potentiometer for position feedback, and a motor control board in one tidy package. This, along with their low cost has made them increasingly popular in the field of robotics. While our method of containing all servos inside the armature allows for Quasi to be quite compact, we are looking at iterating upon the design by moving all servos outside of the character and using sleeved push-pull cables to transfer the mechanical forces from a bank of servo motors to the armature itself.

Quasi has a number of features in addition to his eyelids for conveying emotion, the most prominent of which are LED lighting fixtures for his eyes and antennae. These combine red, green, and blue LEDs to display any color of the spectrum. His antennae can move both forward and backward as well as in and out, giving them an expressive quality not unlike that of a dog's ears. The physical movements of Quasi's eyelids, antennae, and body posture combined with changing LED colors allow him to effectively communicate emotions and personality without the use of speech. Now that silent emotional expression has been achieved, our current research is exploring the use of speech to augment expression. Quasi will always be a work in progress-we have learned a great deal about the strengths and limitations of our design and we hope to improve the technology in future iterations of the figure.

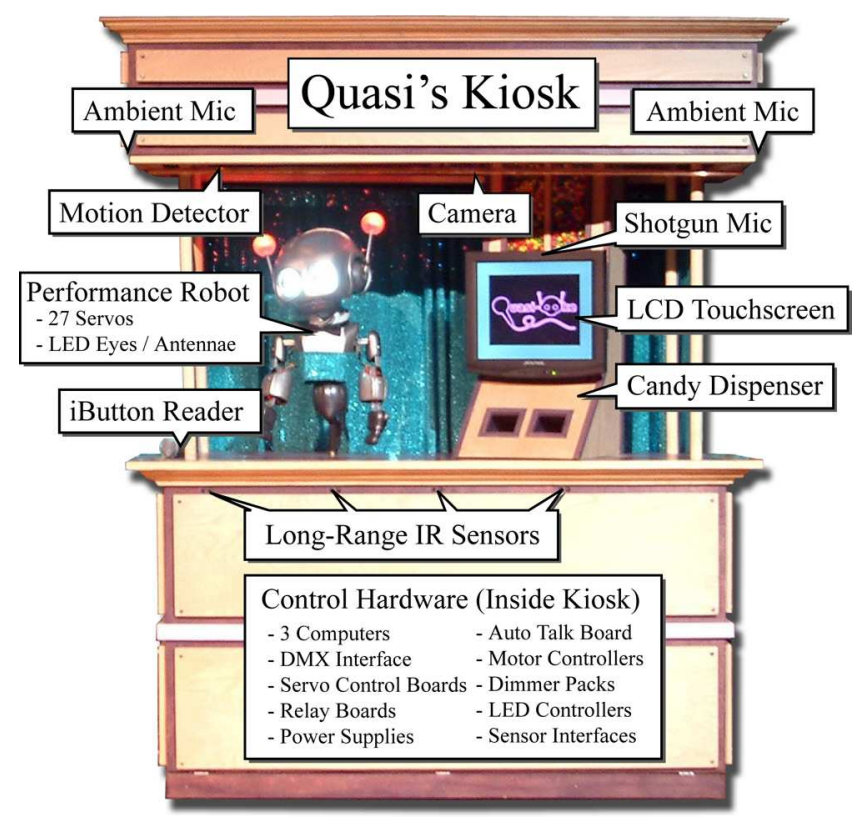

Figure 1: The animatronic character Quasi, his kiosk, and its many sensors.

\subsection{Quasi's Kiosk}

Interactive experiences with the Interbots Platform involve more than just an animatronic figure alone. Quasi's environment - a kiosk that also contains an LCD touch-screen, long range IR sensors, a motion detector, a webcam, stereo speakers, directional microphones, lighting, and a candy dispenser - is just as much a part of the Quasi experience as the robot itself. The kiosk was designed to house Quasi and all of his support equipment in an attractive enclosure that could reside in a hallway at our facilities.

Quasi's torso is tapered down to a pole that allows him to swivel left and right. Combined with whole-body sway movements, he can naturally turn to face and greet visitors. Although we were concerned about people touching Quasi and damaging him, we felt strongly about not having him behind glass or any sort of protective barrier. Without the sharing of a physical space, guests would be deterred from emotionally connecting to the figure. Fortunately, our concerns about damage were unnecessary- unless Quasi holds an arm out as if to shake hands, most guests do not try to touch the figure.

As shown in Figure 1, the kiosk provides information to Quasi through a variety of sensors. A motion detector, identical to those found in home security systems, alerts Quasi to movement in the wide environment. A set of five forwardfacing infrared rangefinders allow Quasi to determine the positions of multiple objects located within five feet of the front of the kiosk. A camera mounted behind Quasi captures video for the face-tracking software, allowing him to direct his gaze towards guests. Stereo microphones mounted on the periphery of the kiosk listen to ambient noise levels and a shotgun microphone picks up speech for voice recognition. An iButton reader allows individuals to $\log$ in and be identified using a low-cost tag carried on keychains. 
Quasi can enhance his environment with sound; stereo speakers mounted in the crown of the kiosk allow him to play speech and music. A nineteen-inch LCD touch screen mounted to the right of the figure allows for intuitive interaction with questions, games, and other active content. Multiple channels of computer-dimmed lighting allow Quasi to manipulate the lights in his kiosk. Most importantly to the residents of our facilities, Quasi's kiosk features an automatic candy dispenser that holds forty-five pounds of Skittles and M\&Ms behind clear plexiglass windows. The candy dispenser, while somewhat frivolous, has proved to be a unique and fun largesse that Quasi can use as an extra incentive to get people to interact with him.

\subsection{The Control Hardware}

Numerous show control components work together to bring Quasi to life, each of them responding to the DMX show control protocol. DMX is most traditionally associated with theatrical and club environments and the control of lighting fixtures, but in recent years its use has exploded to encompass fields as varied as architectural installations and the control of show hardware automation. The protocol is similar to MIDI in that it specifies a set number of serial channels (512) with a corresponding value for each (1-255). DMX devices listen to their assigned range of channels and respond accordingly. In Quasi, a single application (RSCS, discussed in Section 4.1.3) commands a DMX chain that controls not only his movement, but the rest of his kiosk as well. Because of this, we can easily add any of the wide variety of DMX-capable devices on the market today without having to create new data classes or control methods in software.

The source of the DMX data is a USB to DMX interface made by Color Kinetics. Specific devices attached to this interface include power supplies that illuminate the LEDs in his eyes and antennae. Two servo control boards made by Gildefluke support up to thirty-two hobby servos that make Quasi move. A relay board allows us to power Quasi on and off to "put him to sleep". Lighting dimmer packs allow Quasi to set an ambiance and selectively highlight parts of his kiosk. Motor controller boards allow Quasi to turn through the use of a custom closed-loop servo actuation system.

One additional device, an auto-talk board, does not respond to a DMX command. Rather, it is fed a pre-recorded or live line level audio signal and it moves a servo (mounted on Quasi's jaw) in proportion to the signal's amplitude. The net effect is that Quasi appears to speak in time with the audio, without having to invest any time in pre-animating mouth movements.

\section{SOFTWARE}

There are two categories of software used in the Interbots Platform. Section 4.1 discusses the software applications used to control the animatronic character, while the accessible content authoring tools are presented in Section 4.2.

\subsection{Control Software}

\subsubsection{Inter-Process Communication (IPC)}

The Interbots Platform is a distributed system consisting of several large components and a flexible suite of smaller input/output applications. The various processes that comprise an Interbots character communicate to one another via a central message server. This message passing process utilizes the Inter-Process Communication library (IPC) developed in 1994 at Carnegie Mellon University by Reid Simmons for NASA [1]. In the Interbots system, IPC is utilized in a publish/subscribe message model where each sub-application declares to the Central server what types of messages it will send and receive. IPC then routes messages to their appropriate recipients. This method of communication is what allows the Interbots Platform to be quite flexible, supporting a variety of inputs and outputs.

\subsubsection{Character State Control System (CSCS)}

The backend decision mechanism for a character running on the Interbots Platform depends on a set of system inputs and a network of related states and transition rules. Inputs are split into internal "factors" and external "facts." On the backend, this behavioral model is stored in a database format. This behavioral database is accessed in two very different ways by two separate applications, the Behavior Authoring Tool (discussed in Section 4.2.1) and the Character State Control System. The Behavior Authoring Tool (BAT) is the interface through which a content creator develops personalities and behaviors for a character. The Character State Control System (CSCS) is the decision making software that controls the character when it is performing. Effectively, BAT is an offline pre-programming tool while CSCS is an online runtime execution tool.

In order to fully understand BAT, CSCS, and the general theory behind the autonomous behavior available with the Interbots Platform, the terms "fact" and "factor" should be further understood. Facts represent knowledge acquired from the character's external environment. This is typically an input such as sound, video, button presses, IR readings, etc. Facts can be "direct" (e.g. a distance reading from an IR sensor), or they can be indirectly interpreted from an input. An example of an indirect fact might be "Someone's standing in front of me," determined from the direct IR sensor input. Factors represent the character's internal environment. This includes a character's emotional state. For example, the character Quasi has five emotions or motivations (happiness, aggression, loneliness, cleanliness, and attention), each of which is represented on a numerical scale. Factors are the only elements of persistent state that are stored in the behavioral database.

CSCS handles the changing of character state at run time based on a character's behavioral database. An Interbots Platform character's behavioral database is, at the high level, a set of what is called "superstates". These can be thought of as categories of behaviors. The original Quasi personality included Idle, Sleep, Greeting, Farewell, and GiveCandy superstates. An Interbots character will always have a default superstate which is considered the "Idle" state. Each superstate (except Idle) has a set of "entry requirements" or fact and factor requirements that must be true for the character to enter that superstate. If the character's state does not match any superstate entry requirements, CSCS will default to the Idle state.

Superstates also have "stay requirements," or requirements 


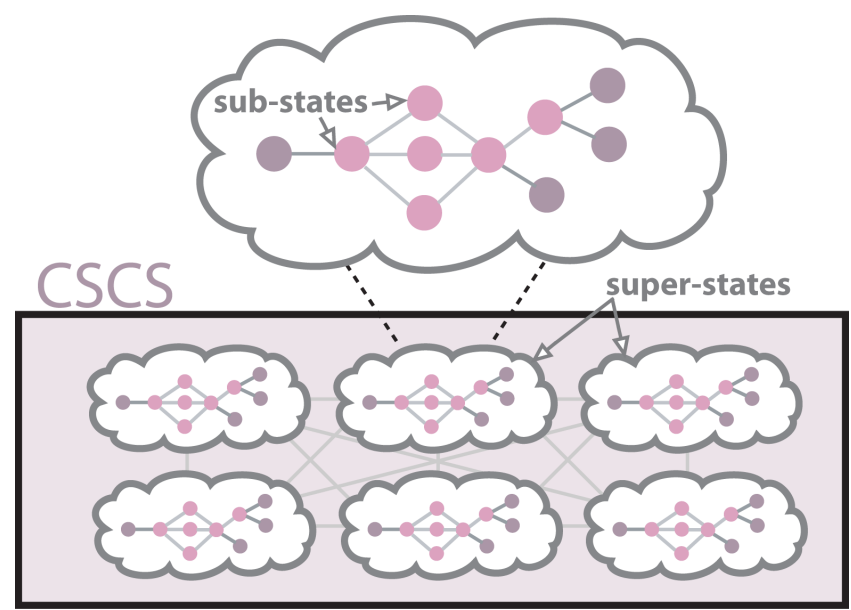

Figure 2: In the Character State Control System, "superstates" are networks of "substates".

that must be true for a character to keep progressing through that superstate. If at any point the character's state fails to meet the stay requirements (or if the character completes a superstate), CSCS will try to find the next appropriate superstate. Superstates are not connected to each other in any explicit way. Whenever CSCS is changing superstates, it considers all possible superstates, finds those whose entry requirements match the character's current state, and then randomly picks one out of those with the highest priority setting.

Within each superstate is a network of substates (see Figure 2). This network can be thought of as a traditional finite state machine. Substates are explicitly connected via "transitions," and the starting substate of each superstate is marked as the "entry substate." At each substate a couple of important things happen. First, adjustments are made to the character's internal factors. Second, "actions" take place. Actions are sequences of output that the character executes. This can include events such as character animations, lighting changes, sounds, candy dispensing, displaying something on a video screen, or even explicitly jumping to a different superstate. Each substate contains a list of these actions, each of which contains a timeline of events. One action is selected from this list and CSCS sends the matching event messages to the output applications through IPC. After a substate is completed, CSCS considers all transitions leading from that substate. If there are no transitions, CSCS exits the current superstate. Transitions can also be weighted to control their probability of being executed and also have transition requirements much like superstates have entry requirements.

\subsubsection{Real-Time Show Control System (RSCS)}

RSCS is the actual software interface to the hardware. It sends DMX data to the servos and plays any authored audio content. RSCS receives messages from CSCS specifying which animation files to load, which channels to play them on, and any sound files that need to be broadcast. It also has the ability to linearly blend animations together, as well as seamlessly transition from one animation to another. An aside to RSCS is the Virtual Show Control System (VSCS), a recent addition which provides a virtual means of previewing the interactive experience in its current state. VSCS provides the same functionality as RSCS, only the end recipient is not a physical character, but rather a virtual 3D animated character running in the open source Panda 3D simulation engine. This provides developers with a simple way to test interactions quickly without the need for a physical character. Content creators can devise interactions even while the physical figure is being constructed or is out of commission.

\subsection{4 cVision}

The Interbots Platform includes a tool for face tracking called "cVision which is an implementation of Intel's OpenCV face tracking library [2]. We believe the ability to detect faces to be a crucial form of input for an interactive character designed to interface with humans. In the Interbots Platform, cVision's face information is factor of highest importance in determining the "point of highest interest for the character. This point then influences where the character "looks, i.e., its gaze target. For Quasi, this gaze target is allowed to override control of his neck, allowing him to appear to be looking directly at the face of a guest. The variation in movement induced by this method also lends an element of realism and believability to the character's movement.

\subsubsection{MIDI Reader, iButton Reader, and Babble}

In order for Quasi to perceive his environment, he must be able to read values from his sensors. The MIDI Reader application listens to values streaming in from a custom circuit board that translates analog voltages to MIDI. It then translates the MIDI signals to IPC messages and passes them to CSCS.

The iButton Reader application interfaces with a low-cost iButton reader module over RS-232. Residents in the building carry iButton tags on their key chains, which when pressed to a reader on Quasi's kiosk allow him to identify specific people and greet them accordingly. iButtons provide a unique identification number that can be correlated to a user database. Their greatest benefit is the low-cost and ruggedness of the tag and reader, critical when people carry one with them at all times.

The Babble application is a simple implementation of the open-source Sphinx voice recognition package developed at Carnegie Mellon University, enhanced with the capability to pass messages over IPC. Babble identifies phonemes which are then matched against a database of predefined words, and any matches are passed to CSCS over IPC for implementation. This system works fairly well for simple voice responses to Quasi's questions.

\subsection{Content Authoring Tools}

One of the goals of the Interbots Initiative was to create content authoring tools that are so intuitive and easy to use that no prior programming or technical skills are required. This was accomplished by leveraging the power of popular, mass-market software packages, as well as creating custom, graphical authoring tools, allowing developers to quickly and comfortably author original interactive content for a animatronic character. 


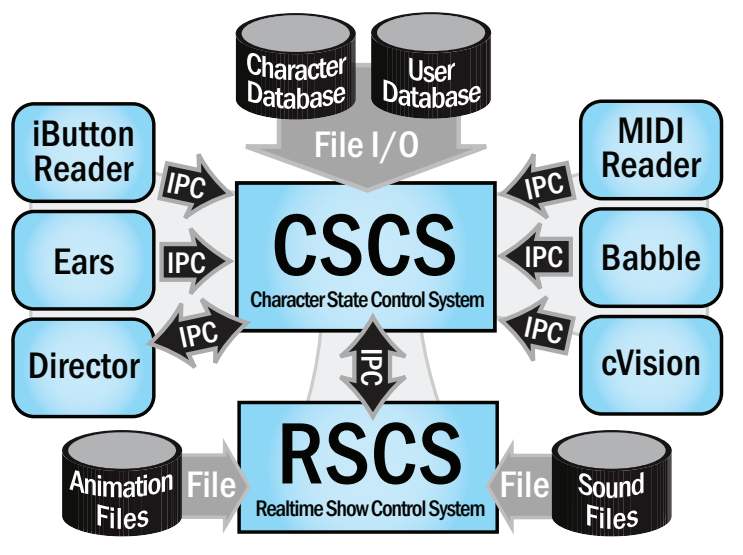

Figure 3: An overview of the Interbots Platform control software.

\subsubsection{Behavior Authoring Tool (BAT)}

One of the great strengths of the Interbots Platform is the Behavior Authoring Tool (BAT). Used to author content prior to run-time, BAT is an application for composing networks of superstates and substates (see Section 4.1.2). BAT provides an intuitive graphical interface to manipulate the data in the behavior database, allowing non-programmers to easily create character personalities and script all character behaviors. Currently BAT is an application written in Macromedia Flash that interfaces with the character database by posting SQL commands through an ODBC-PHP connection. BAT provides a visualization of each superstate, making it easy to understand the complete picture of a character's personality.

\subsubsection{Director}

One of the most powerful means of interacting with Quasi resides in Macromedia Director, the popular tool for authoring multimedia content. It comes with a powerful scripting language and support for many media types, and its graphic abilities provide a perfect means for visualizing material. Splash screens, touch screen interfaces, video playback modules, interactive games- all of these can be easily created in Director. We developed a custom plug-in to allow it to communicate with the rest of the Interbots Platform, thereby allowing information to pass easily from the touch screen to the CSCS control application.

\subsubsection{Maya Plug-In}

Alias Maya is the industry standard application for 3D modeling, animation, and effects, used by professionals and students alike. It seemed the natural choice for opening up animation of the animatronic characters to 3D animators. To this end, we created a pipeline for exporting animations from Maya to the physical robot. Moreover, the pipeline is not limited to merely joint rotations, but can also control light levels and colors, within both the figure and its environment. In fact, anything that can be controlled by DMX can be easily added to the pipeline, provided it can be tied to some keyable attribute in Maya. The pipeline, as it stands, consists of three parts: 1) A template Maya file of the current robot with appropriately-limited joints and controls, 2) A servo setup configuration file listing all

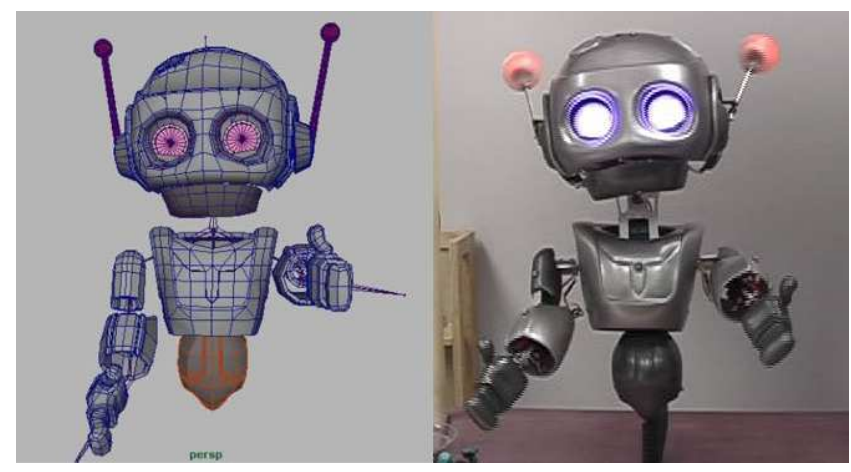

Figure 5: Animations developed using the one-toone Maya model are easily exported to the animatronic character's control system.

servos and lights and their associated controls, and 3) The MEL script, a simple tool that exports animations to any of the desired DMX channels, converting keyframes to the appropriate DMX values

Additionally, we created an Animation Editor Tool (AET) for those uncomfortable animating in the Maya environment. The AET essentially consists of a series of sliders for controlling the figures joints and lights (see Figure 4). The Maya Plug-in and AET output a file containing DMX signal values for selected servos. As the file is read by the RSCS application (Sec 4.1.3), the signal values are sequentially sent to the servos, animating the character in a lifelike manner (see Figure 5).

\section{RAPIDLY DEVELOPED INTERACTIVE EXPERIENCES}

For years the ability to create and program humanoid robots has fallen into two distinct camps- the researchers at universities studying human-robot interaction or artificial intelligence, and the entertainers designing animatronic shows at venues like Disney Theme Parks. With the advent of domestic entertainment robots, hobbyists have expressed desire to join in the foray. The development of an easy to use, extensible platform for content creation for these domestic robots will open the doors to artistic exploration of this medium. As demonstrated by the students in Carnegie Mellon University's Building Virtual Worlds (BVW) course, the Interbots Platform may soon allow this desire for exploration to become a reality.

The usability of the Interbots Platform's content authoring tools was put to the test when students in the Fall 2004 BVW course had the opportunity to develop interactive experiences for Quasi in two-week project cycles. The tools met expectations admirably- interdisciplinary teams of four students (one programmer and three artists, none of which had prior experience with the Interbots Platform), were able to easily and rapidly develop the following completely original interactive experiences.

\subsection{Quasi's Photo Kiosk}

When guests visit the ETC, they love to have their picture taken with Quasi. Until November 2004, these pictures 


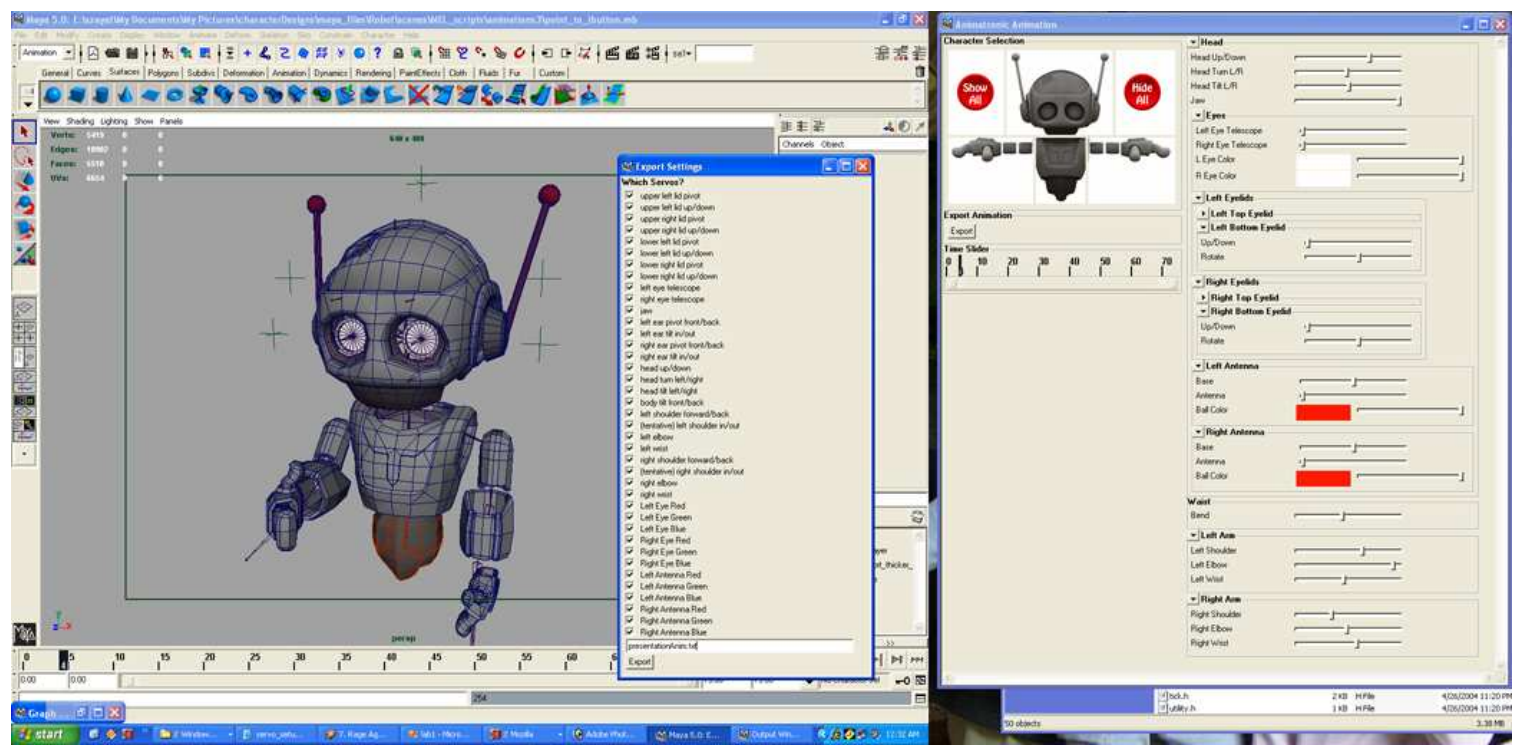

Figure 4: The Maya Plug-In allows artists to easily author animations for the animatronic character.

(often taken with celebrities) have resided on personal computers, inaccessible to the public. After visiting the Kodak Imageworks Lab at Disney's Epcot Theme Park, the BVW team that worked on this experience was inspired to create a photo album for Quasi. The album contains pictures of his friends and family, as well as a short illustrated story about how he was created. The picture taking process was also automated. The team set up a USB camera across from Quasi's kiosk and wrote a program that took a guest's picture with Quasi, e-mailed the picture to them, and then automatically added the picture to the photo album. While guests peruse the photo album, Quasi reacts to many of the pictures, and even does impressions of the celebrities in the photos.

\subsection{Quasi's Interactive Dreams}

Unlike the show animatronics found in permanent entertainment installations, Quasi's hobby-grade servo motors aren't designed to withstand continuous operation. The BVW team that worked on this experience addressed the problem of how to keep Quasi engaging, entertaining, and interactive while allowing his motors to "rest." Their solution was to create interactive dreams for a "sleeping" Quasi. Displayed on Quasi's touchscreen, the dreams can be happy, neutral, or scary. Quasi will twitch and his eyes and antennae will change color in response to the content of a dream. If a guest approaches Quasi while he's dreaming, he will wake up. If approached while having a happy dream, Quasi will wake angry, but if approached while having a nightmare, Quasi will wake happy and relieved. While some of the dreams are purely visual, others are interactive touchscreen games, allowing the guest to guide the direction of the dreams.

\subsection{Quasi-oke}

In "Quasi-oke", the first theatrical piece starring Quasi, a "volunteer" from the audience is asked to come up to Quasi's kiosk and select a song to sing. The volunteer selects the duet "Summer Nights" from the musical Grease. After she convinces a bashful Quasi to sing the duet with her, Quasi asks his friends (C3PO, R2D2, Hal, and AIBO) to sing the male backup and requests that the audience sing the female backup. This 10 minute musical/comedy piece was the finale of the Fall 2004 Building Virtual Worlds Show, a spot typically reserved for the best piece of the semester.

\section{FUTURE WORK}

While the current system allows for simple and rapid content development, its framework is inherently tied to the hardware configuration. The next step for the Interbots Initiative is to generalize this environment to accommodate different characters and different inputs. The goal is to be able to attach an entirely new set of hardware to the control system, author content for it, and interact with it, all without having to rewrite and recompile any code. This involves generalizing the message system used for inter-program communication, creating a mechanism to inform the control programs of the hardware configuration, and modifying the authoring system so that it can create new configurations from a pool of available hardware.

The Behavior Authoring Tool is being redesigned to accommodate the new abstracted system of configuration files. It is also being expanded to allow users to define any number of custom inputs, variables, and outputs that they wish to incorporate into the robot. It will become possible to author content that can respond to unknown devices and send custom output to any device that is listening.

As an augmentation to the CSCS system, a Guided Performance Interface (GPI) is being developed. The GPI provides access to all of a character's sensors and actuators, allowing a performer to virtually puppeteer the character. The user will be able to control low level functions like firing an animation or playing a sound, as well as manipulate higher level actions such as dictating where the character's attention is focused or modifying the valence and intensity of its emotions. Running on a wireless Tablet PC, the graphical user interface will include a real-time 3D rendering of the robot, 
live streaming video feeds that allow the performer to see through the robot's cameras, as well as variable and sensor data shown in easy to read graphical formats. The graphical tool will allow the user to drag and drop components to perform simple actions. More complicated control will be achieved with a standard gamepad-type joystick, giving the performer the ability to puppeteer the robot continuously and fluidly in response to live events. To further enhance the real-time performance, the GPI will incorporate a microphone that will transmit the puppeteer's voice to the Platform's sound system. The aforementioned autotalk board (Section 3.3) will allow the robot to synch its mouth to an enhanced version of the puppeteer's voice, allowing the puppeteer to speak through the robot in real-time.

These expansions of the Interbots Platform will further magnify its extensibility, allowing content developers to author interactive experiences using any subset of available hardware and create custom inputs and outputs for the system. The Platform also brings another type of artist-the performer-into the system, allowing an animatronic character to be fully puppeteered in real-time.

\section{CONCLUSIONS}

The Interbots Initiative follows the philosophy of technological transparency: allow technology to support the experience, but not become the experience. While much of the work we have done is highly technical, our end goal is a solid and engaging experience. We do not implement technology for technology's sake, and would rather not support a feature or capability if we feel it does not contribute to the character we are trying to create. Guests should forget they are interacting with an autonomous hunk of wires, metal, and plastic. They should see the character in front of them, not the robot. No matter how impressive and complicated the technology becomes, if a believable personality has not been created then an audience will not be fully engaged.

The flexibility of the Interbots Platform allows designers to explore animatronic expression of personality and emotion without the construction of a single mechanical component. $3 \mathrm{D}$ artists constructed a virtual prototype of the animatronic character, experimented with movement by creating and running animations on the prototype, and then refined the design. In this iterative manner, a communicative, expressive, and realistic character design was finalized. The primary contribution of the Interbots Platform is that it provides an interface between artistic vision and technological implementation.

With a character in place, attention was focused to creating a system of authoring tools that allows for rapid development of content. Content is a vital part of any platform and should not necessarily be handled by the same people who designed and created the mechanical components. Realizing this, we created a suite of tools that would not only be accessible to technical experts, but to non-technologists such as writers and artists as well. We leveraged the power of Maya and Director, two applications most artists are very familiar with, to create the primary content authoring tools for animation and interactivity. For the more complex task of programming Quasi's artificial intelligence, we developed an intuitive graphical authoring tool in Flash. Combined, these tools allow practically anyone to create a Quasi experience in a very short period of time. This was actually put to the test when Quasi was used in the Building Virtual Worlds course at Carnegie Mellon University. Interdisciplinary teams of four students who had no prior experience with the Interbots Platform were able to develop new and original content for Quasi in two-week development periods.

\section{ACKNOWLEDGMENTS}

The authors wish to acknowledge the following members of the Spring 2005 Interbots Initiative Team for their continued work on this project: William Bosley, Dave Culyba, TJ Jackson, Shane Liesegang, Christine Skarulis, and Jim Valenti.

\section{ADDITIONAL AUTHORS}

Peter Stepniewicz (The Entertainment Technology Center, email: pstepnie@andrew.cmu.edu), Salim Zayat (The Entertainment Technology Center,

email: szayat@andrew.cmu.edu), Jichen Zhu (Georgia Institute of Technology, email: gtg405r@mail.gatech.edu), Seema Patel (The Entertainment Technology Center, email: seemap@andrew.cmu.edu), and Brenda Harger (The Entertainment Technology Center, email: bharger@andrew.cmu.edu).

\section{REFERENCES}

[1] http://www-2.cs.cmu.edu/afs/cs/project/TCA/www/ ipc/ipc.html.

[2] http://isa.umh.es/pfc/rmvision/opencvdocs/.

[3] 2002. http://www.etc.cmu.edu/projects/iai/.

[4] 2002. http://disneyworld.disney.go.com/wdw/ entertainment/entertainmentDetail?id= LuckytheDinosaurEntertainmentPage\&bhcp $=1 \&$.

[5] C. Breazeal, A. Brooks, J. Gray, M. Hancher, J. McBean, W. Stiehl, and J. Strickon. Interactive robot theatre. Communications of the ACM, 46(7):76-85, 2003.

[6] C. Breazeal, A. Brooks, J. Gray, G. Hoffman, C. Kidd, H. Lee, J. Lieberman, A. Lockerd, and D. Mulanda. Tutelage and collaboration for humanoid robots. International Journal of Humanoid Robotics, 1(2):315-348, June 2004.

[7] A. Bruce, I. Nourbakhsh, and R. Simmons. The role of expressiveness and attention in human-robot interaction. In Proceedings of the IEEE International Conference on Robotics and Automation. ICRA '02, May 2002.

[8] H. Greiner, 2004. Stated in her talk at the RoboNexus Conference on October 21.

[9] J. Pineau, M. Montemerlo, M. Pollack, N. Roy, and S. Thrun. Towards robotic assistants in nursing homes: Challenges and results. Robotics and Autonomous Systems: Special issue on Socially Interactive Robots, 42(3-4):271-281, 2003. 